\title{
One month of the novel coronavirus 2019 outbreak: Is it still a threat?
}

\author{
Apu Chowdhury ${ }^{1} \cdot$ Nabila Jahan $^{1} \cdot$ Sicen Wang $^{1}$
}

Received: 29 February 2020/Accepted: 1 April 2020/Published online: 20 April 2020

(C) Indian Virological Society 2020

\begin{abstract}
The novel coronavirus 2019 disease (COVID19) is now in an outbreak not only in China but also around the world, suspected to be originated from a wet market in Wuhan, Hubei province, China. The flare-up of COVID19 , it has already been infected 78,811 people with 2462 fatalities in 1 month window. The most alarming issue is the virus can transmit from host to host and still asymptomatic. Currently, 24 counties with 505 confirmed cases have been reported. Presently, there is no specific treatment or vaccine but physicians are battling with the use of antibiotics, steroid, anti-viral and anti-HIV drugs and some of the infected cases are testified improved. WHO and China National health Commission are cooperatively striving to come up with elucidation but it will take a minimum of 3 to 4 months at least to undergo a phase- 1 trial. However, the soaring rates of spreading each day has become much stable which might even improve within the next few weeks in China but not for some other countries. Healthy peoples are instructed to avoid public gathering, always wearing the mask and frequently wash hands. Currently, China has able to hold up more than $97.7 \%$ infection within China but a scientific breakthrough is crucial before it's too late.
\end{abstract}

Keywords COVID-19 · Novel coronavirus 2019 · Wuhan · SARS-CoV-2

Apu Chowdhury

dr.apu05@gmail.com

1 School of Medicine, Xi' an Jiaotong University, Xi'an 710061, China

\section{Introduction}

Coronaviruses $(\mathrm{CoV})$ are enveloped viruses with a singlestranded RNA genome. CoV ranges from 26 to 32 kilobases which makes it the largest RNA virus. CoVs are of four kinds which are alpha, beta, delta, and gamma. Among these types, only alpha and beta $\mathrm{CoV}$ can infect humans. In 2003 Severe Acute respiratory syndrome CoV (SARS$\mathrm{CoV}$ ) and 2012 Middle East respiratory syndrome CoV (MERS-CoV) caused the global epidemic. From 2003 to 2004 SARS-CoV infected 8096 people in 29 countries where 774 souls were lost [22]. MERS-CoV was even deadly and took 858 lives out of 2494 people since 2012 [23]. Even so in 2019, the latest strain was first identified in Wuhan (Hubei province, China) suspected to be a novel coronavirus 2019 (SARS-CoV-2) in the same family of $\mathrm{CoV}$ and not the unique factor in this virus is animal to human transmission which already infected 78,811 people and 2462 lives expired within 1 month (January 21th to February 23rd 2020) [24]. The amount of death and infection already passed SARS-CoV and MERS-CoV infection where, apart from China, 28 other countries by now detected COVID-19 and most of those confirmed cases had a travel history to Wuhan and due to asymptomatic behavior SARS-CoV-2 was able to transmit more human to human.

\section{Current understanding}

SARS-CoV-2 belongs to the Beta coronavirus group which is enveloped, single-stranded RNA and able to infect herbs, animals and humans. Bats are being suspected for the SARS-CoV-2 host but there were likely to be involvement of some intermediate host in bat-human transmission. S-protein of SARS-CoV-2 interacts with human 
Angiotensin-converting enzyme 2 (ACE2) but they have genetics distance from SARS-CoV even more than MERSCoV [27]. SARS-CoV-2 has 380 amino acid replacements from SAR-CoV and SARS-like coronavirus [26].

By January 2nd, 2020, 41 patients were admitted with COVID-19 where all of them exposed to the Huanan seafood market. This COVID-19 was detected by performing real-time PCR from the lower respiratory tract specimen. All of these 41 patients had fever and pneumonia with abnormal findings in chest CT scans. More severe patients have higher plasma levels of IL2, IL7, IL10, GSCF, IP10, MCP1, MIP1A, and TNFa. Novel COVID-19 have some similar symptoms to SARS (e.g. fever, dry cough, shortness of breath) but there are some important differences, such as the absence of upper respiratory tract symptoms (rhinorrhea, sneezing, and sore throat) and intestinal symptoms, such as diarrhea [6]. Another study showed that a healthy 6 membered family from Shenzhen traveled to Wuhan in between December 29th, 2019, to January 4th, 2020, where 5 members were diagnosed with COVID-19. All of these 5 patients showed upper and lower respiratory tract symptoms, fever, and diarrhea [2]. In between December 8th, 2019 to February 6th, 2020 only nine infants were diagnosed with SARS-CoV-2 positive and none of them had to undergo intensive care unit (ICU). Eight of these nine patients stayed in Wuhan or one of their family member visited Wuhan which again confirms the human to human transmission [21]. Human to human transmission is confirmed with this new virus [11] and the basic reproductive number $\left(\mathrm{R}_{0}\right)$ was estimated at 2.2 [8]. Despite the lack of evidence of super spreader, at this $R_{0}$ rate, this epidemic could be the most alarming health situation for China.

\section{Present COVID-19 management}

Scientists found coronaviruses are challenging to replicate in the laboratory which makes it more repressing to create cures or vaccines. Most viruses can mutate quickly and makes them easier to catch and spread, one of the reasons a person can catch a cold over and over again. Disproportionately, this attack is vulnerable to the population with a weak immunity system. Despite $70 \%$ genetic similarities with SARS-CoV, the novel COVID-19 also have some dissimilar symptoms from SARS-CoV [7]. But due to its novelty and time pressure still, there is no appropriate treatment developed. Scientists at the National Institute of Allergy and Infectious Diseases Vaccine Research Center have used nucleic acid vaccine platform approaches to create vaccines for SARS-CoV or MERS-CoV, which are also being pursued. Using messenger RNA (mRNA) vaccine technology may accelerate to finding a cure [10].
Real time-PCR is the current method to detect COVID19. In China, BGI group (a genome sequencing company) established an emergency lab in Wuhan which can test 10,000 samples daily. WHO also dispatched 250,000 COVID-19 testing kit among 159 laboratories around the world [15]. SARS-CoV-2 viral load is more consistent in upper respiratory track specimen [28]. A study of 8274 COVID-19 samples suggested that nasopharyngeal swabs gave more consistent results than sputum. In this study they evaluated a fluorescence based RT-PCR distributed by Chinese Center for Disease Control and Prevention but also suggested that a more accurate testing kit is necessary to get precise detection [19]. In this urge whole world is trying to come up with a better solution as soon as possible.

In Jin Yin-tan Hospital (Wuhan, China), COVID-19 diagnosed patients received antibiotics (oral and intravenous) and Oseltamivir (orally $75 \mathrm{mg}$ twice daily) side by side, Corticosteroid therapy (methylprednisolone 40$120 \mathrm{mg}$ per day) was given as a combined in case of severe pneumonia. Severe hypoxemia patients received Oxygen support (e.g., nasal cannula and invasive mechanical ventilation) [6]. Shanghai Institute of Materia Medica and Shanghai University of Science and Technology mutually found a group of old medicines and traditional Chinese medicines (TCM) that might present a therapeutic effect on COVID-19. They have suggested protease inhibitors indinavir, saquinavir, lopinavir, carfilzomib, ritonavir, and 12 other anti-HIV drugs, 2 anti-respiratory syncytial virus drugs, 1 anti-human macrophage drug, 1 anti-schizophrenia drug, 1 immunosuppressive agent and 2 old drugs cinnamyl amine and cyclosporine. They also recommended that Chinese herbal medicines such as, polygonum cuspidatum and shandougen (Table 1) possibly have an active ingredient that can fight against COVID-19 [14]. Another study indicates that the use of corticosteroid might not improve COVID-19 treatment because it can suppress the immune response and pathogen clearance besides improving lung inflammation [13]. A study of 138 patients in Zhongnan Hospital of Wuhan University with COVID-19 positive was treated with anti-viral (oseltamivir), antibacterial (moxifloxacin, ceftriaxone, azithromycin) and Glucocorticoid therapy. Most interestingly $6(4.3 \%)$ died and 47 (34.06\%) patients were discharged with 10 days of median hospital stay. Out of 138 patients 40 health professional were infected with COVID-19 which confirms nosocomial infection capability of SARS-CoV-2 [17]. The first case COVID-19 in the USA has been recovered with the use of remdesivir (GS-5734), a novel nucleotide analog prodrug that is under development. In this case report, the patient was treated with vancomycin and cefepime from day 5 of hospitalization (9th day of illness) but no improvement and at the day 7 (11th day of illness) of these drugs were discontinued and remdesivir was introduced. The very next 
Table 1 The list of antiviral, antibiotics, steroids, traditional Chinese medicine (TCM) and other drugs which includes immunosuppressants*, antibacterial $^{\# \#}$, antimalarial ${ }^{\#}$ and antirheumatic** drugs were wither currently in practice or suggested for COVID-19 managements

\begin{tabular}{llr}
\hline Antiviral & Oseltamivir, remdesivir, $\alpha$-interferon, lopinavir+ritonavir, ribavirin & In practice \\
& Indinavir, saquinavir, lopinavir, carfilzomib, ritonavir, redcivir, favipiravir & Suggestion \\
Antibiotics & Moxifloxacin, ceftriaxone, azithromycin & In practice \\
Steroids & Methylprednisolone, Glucocorticoid & In practice \\
TCM & Polygonum cuspidatum, shandougen & Suggestion \\
Other & Cyclosporine*, baricitinib**, chloroquine phosphate ${ }^{\#, \text { Cinnamyl amine }}$ & Suggestion \\
\hline
\end{tabular}

day (day 8) patient health conditions became much better. He was afebrile and all symptoms improved except mild cough side by side his viral load also lowered to $\mathrm{Ct}$ (real time PCR gene expression) 37-40 which was Ct 18-20 on day 4 of his hospitalization [5]. Another in vitro (Vero E6 cells) study suggested the combination of remdesivir and chloroquine could be a choice. [18] This study indicates the use of remdesivir could be a major breakthrough for the treatment of COVID-19. Due to the lack of large clinical data, mass application of remdesivir still not elucidated. Another suggested drug was baricitinib, which is known for its rheumatoid arthritis treatment. Baricitinib can also inhibit AP2-associated protein kinase 1 and cyclin G-associated kinase which are important tools of viral endocytosis [12].

According to the Chinese clinical trial registry (a database of biomedical studies in China) there are now more than 200 clinical trials are conducting on different kinds of antiviral, TCM and stem cell for better managements of COVID-19. But if standard study protocol like control groups, clinical outcomes and randomization are not followed then these clinical trials would be questionable. National Health Commission and National Administration of Traditional Chinese Medicine gave guidance for COVID-19 management in the Fifth Edition for Trial Implementation titled as "Diagnosis and treatment protocol of novel coronavirus pneumonia infection". Here they suggested using $\alpha$-interferon atomization inhalation (5 million $U$ or equivalent dose, added with $2 \mathrm{ml}$ sterile injection water, twice daily for adults), Lopinavir/Ritonavir (200 mg/50 mg per pill) 2 capsule each time, twice a day and also Ribavirin can be added (500 $\mathrm{mg}$ each injection, I. V. two to three times a day). Some other antiviral drugs like Redcivir, Chloroquine Phosphate, and favipiravir also suggested but strongly advised not to use broad spectrum antibiotics unnecessarily [3].

\section{Infection status after 1 month of the outbreak}

WHO initiated meticulous monitoring by posting a daily update on the total number of infection cases and death by the COVID-19 since January 21th, 2020. By analyzing these data, major outbreak has been contained $(97.76 \%)$ inside China though there are 1769 cases reported around 28 countries; however, South Korea (602) Japan (132), Singapore (89), and Italy (76) are now most vulnerable. Till February 23th a total number of 78,811 cases are enlisted where China alone contained 77,042 cases. A total number of 2445 death (3.1\% of total cases) were recorded which mostly came from Hubei province (2346) and globally seventeen were deceased with this infection [24]. Within 33 days (21th January to 23th February, 2020) the infection has been increased 279 times which made this COVID-19 even more dangerous than SARS-CoV or MERS-CoV. According to the data 5th February daily new cases (Fig. 1a) hit the peak (3924) where at 12th February we saw the most deaths (254) in 1 month of COVID-19. There was a sharp total cases spike in between 16th and 17th February (Fig. 1c) because China was started to report new cases on the basis of clinical and laboratory confirmation. According to CCDC (China Center for Disease Control) 22,888 patients were recovered and discharged but currently, 628,517 close contacts have been followedup with, and 106,089 are still under medical observation till February 24, 2020 [1]. By February 11, 2020 in 422 medical facilities serving COVID-19 patients, a total of 3019 health workers have been infected (1716 confirmed cases), and 5 have died [16]. To reduce nosocomial transmission health care personals were advised to wear N95 mask under personal protective equipment and also the operation theater should be operating under negative pressure [20].

\section{Precautions for the uninfected}

According to the WHO, this contamination is spreading with human to human contact, droplets, and fomites. So it has been recommended that one should always wear a mask and wash hands frequently, especially after direct contact with ill people. People are recommended to avoid direct contact with persons have severe respiratory syndromes and sidestep mass people gathering [24]. Infants can't wear mask, so special care protective measures are required which includes wearing protect masks by adult 

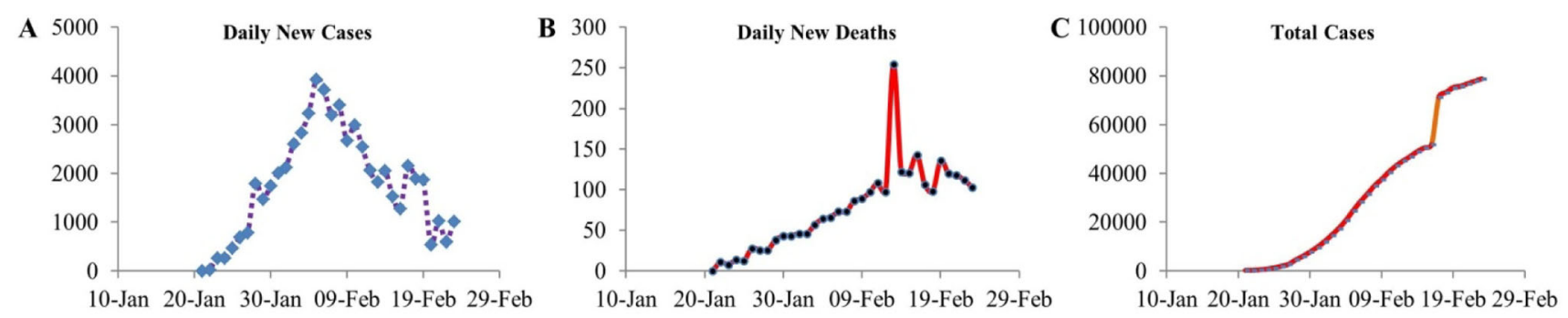

Fig. 133 days (Jan 21th to Feb 23th, 2020) epidemic status of COVID-19 where a represents daily new cases, b shows daily new death, c illustrate total new cases

caretakers, washing hands before coming close contact and continuous sterilization of the toys and surface [21]. People with a good immunity system might have a better chance of survival. So, immune-boosting foods and supplements might be effective [9].

\section{Discussion}

At the time of writing this article, the Chinese central government prohibited all kinds of transport in and out of Wuhan. At the train station, airport and bus station they installed a thermal sensor to detect the body temperature of the passengers. Each community in every city has taken special quarantine protocols. In this protocol, only one member of each family is allowed to go out once in a 2 to 3 days. Each time of outing everyone has to write down their detailed information in a logbook and while at return their body temperature has been measured and noted. Nevertheless, the major problem lies with the asymptomatic behavior of potential carriers which insinuate it doesn't show any symptoms within 2 to 10 days of invasion inside a host and still can spread the contamination [25]. Purportedly, infection transpires when someone doesn't have any noticeable symptoms which make it more threatening when comes to spreading controlling and identifying. The Chinese government already extended this year's spring festival holidays, so people can have time to combat with the current situation. It's a real irony that a couple of scientists from Wuhan Institute of Virology (February 2019) published an article which forecasted that China would likely be the next possible hotspot of coronavirus outbreak. Popular cultural beliefs in slaughtering the live animal to consume more nutrition and close contact with the bats were suspected for the potential reemerge of the viral transmission [4].

Current treatment was able to discharge lots of COVID19 infected patients after two nucleic acid tests were negative which means there were no virus replication. At the same time these recovered patients produced their own antibody which can last longer than 6 months so, second degree infection is unlikely unless there is a virus mutation [3]. WHO and China National health Commission is trying to design specific vaccines by isolating the virus from the host cells. China's government took very cautious action immediately by sharing the gene sequence with the WHO to facilitate the vaccine invention to accelerate the assessment. Plasma transfusion from the recovered patients might show promises because the recovered patients already developed antibodies to fight against COVID-19 which could help to replicate same immunity to the infected ones.

Nonetheless, all viruses are different and none knows international boundaries but it's a very early stage to project the trajectory of this novel $\mathrm{CoV}$ outbreak. Broadspectrum antiviral like remdisivir, chloroquine phosphate, favipiravir, oseltamivir, $\alpha$-interferon, lopinavir+ritonavir, ribavirin possibly present a light of hope on the novel COVID-19 management side by side with the proper public health strategies also very important to contain this outbreak.

\section{Compliace with ethical standards}

Conflict of interest The authors declared that there is no conflict of interest.

\section{References}

1. Abduljalil JM, Abduljalil BM, Epidemiology, genome, and clinical features ofthe pandemic SARSCoV-2: a recent view. New Microbes New Infect. https://doi.org/10.1016/j.nmni.2020. 1006721 .

2. CCDC. Tracking the epidemic. 2020.

3. Chan JF, Yuan S, Kok KH, et al. A familial cluster of pneumonia associated with the 2019 novel coronavirus indicating person-toperson transmission: a study of a family cluster. Lancet. 2020;395 (10223):514-23. https://doi.org/10.1016/S0140-6736(20)301549.

4. CCDC. COVID-19 prevention and control. CCDC 2020.

5. Čivljak R, Markotić A, Kuzman I. The third coronavirus epidemic in the third millennium: what's next? Croatian Med. J. 2020;61(1):1-4. https://doi.org/10.3325/cmj.2020.61.1.

6. Fan Y, Zhao K, Shi ZL, Zhou P. Bat Coronaviruses in China. Viruses. 2019;11(3):210. https://doi.org/10.3390/v11030210. 
7. Holshue ML, DeBolt C, Lindquist S, Lofy KH, Wiesman J, Bruce $\mathrm{H}$, et al. First case of 2019 novel coronavirus in the United States. N Engl J Med. 2020.

8. Huang C, Wang Y, Li X, et al. Clinical features of patients infected with 2019 novel coronavirus in Wuhan. China. Lancet. 2020;395(10223):497-506. https://doi.org/10.1016/S0140-6736 (20)30183-5.

9. Hui DS, Azhar E, Madani TA, Ntoumi F, Kock R, Dar O, et al. The continuing 2019-nCoV epidemic threat of novel coronaviruses to global health-the latest 2019 novel coronavirus outbreak in Wuhan, China. Int J Infect Dis. 2019;2020(91):264-6.

10. Li Q, Guan X, Wu P, et al. Early transmission dynamics in Wuhan, China, of novel coronavirus-infected pneumonia. N Engl J Med. 2020;382(13):1199-207. https://doi.org/10.1056/NEJ Moa2001316.

11. London C. 14 Functional foods that boost the immune system. Funct Food Product Dev. 2010;2:295.

12. Paules CI, Marston HD, Fauci AS. Coronavirus infections-more than just the common cold. JAMA. 2020. https://doi.org/10. 1001/jama.2020.0757.

13. Phan LT, Nguyen TV, Luong QC, et al. Importation and humanto-human transmission of a novel coronavirus in vietnam. N Engl J Med. 2020;382(9):872-4. https://doi.org/10.1056/NEJMc200 1272.

14. Richardson P, Griffin I, Tucker C, et al. Baricitinib as potential treatment for 2019-nCoV acute respiratory disease. Lancet. 2020;395(10223):e30-1. https://doi.org/10.1016/S0140-6736(20) 30304-4.

15. Russell CD, Millar JE, Baillie JK. Clinical evidence does not support corticosteroid treatment for 2019-nCoV lung injury. Lancet. 2020;395(10223):473-5. https://doi.org/10.1016/S01406736(20)30317-2.

16. science Cao. 上海药物所和上海科技大学联合发现一批可能对 新型肺炎有治疗作用的老药和中药. 2020.

17. Team. TNCPERE. The epidemiological characteristics of an outbreak of 2019 novel coronavirus diseases (COVID-19) China. 2020.

18. Wang D, Hu B, Hu C et al. Clinical characteristics of 138 hospitalized patients with 2019 novel coronavirus-infected pneumonia in Wuhan, China. JAMA. 2019;2020:e201585. https://doi.org/10.1001/jama.2020.1585.

19. Wang M, Cao R, Zhang L, et al. Remdesivir and chloroquine effectively inhibit the recently emerged novel coronavirus (2019nCoV) in vitro. Cell Res. 2020;30(3):269-71. https://doi.org/10. 1038/s41422-020-0282-0.

20. Wang M, Wu Q, Xu W, Qiao B, Wang J, Zheng H, et al. Clinical diagnosis of 8274 samples with 2019-novel coronavirus in Wuhan. https://doi.org/10.1101/2020.02.12.20022327

21. Wax RS, Christian MD. Practical recommendations for critical care and anesthesiology teams caring for novel coronavirus (2019-nCoV) patients. Can J Anaesth. 2020. https://doi.org/10. 1007/s12630-020-01591-x.

22. Wei M, Yuan J, Liu Y, Fu T, Yu X, Zhang Z. Novel Coronavirus Infection in Hospitalized Infants Under 1 Year of Age in China. JAMA. 2020;323(13):1313-4. https://doi.org/10.1001/jama.2020. 2131.

23. WHO. Summary of probable SARS cases with onset of illness from 1 November 2002 to 31 July 2003, Geneva. 2003.

24. WHO. Middle East respiratory syndrome coronavirus (MERSCoV). 2019.

25. WHO. Coronavirus disease 2019 (COVID-19) situation Report34. 2020.

26. WHO. Novel Coronavirus (2019-nCoV) situation reports. 2020.

27. Xu X, Chen P, Wang J, et al. Evolution of the novel coronavirus from the ongoing Wuhan outbreak and modeling of its spike protein for risk of human transmission. Sci China Life Sci. 2020;63(3):457-60. https://doi.org/10.1007/s11427-020-1637-5.

28. Zou L, Ruan F, Huang M, et al. SARS-CoV-2 Viral Load in Upper Respiratory Specimens of Infected Patients. N Engl J Med. 2020;382(12):1177-9. https://doi.org/10.1056/NEJMc2001737.

\section{Publisher's Note}

Springer Nature remains neutral with regard to jurisdictional claims in published maps and institutional affiliations. 